\title{
INITIAL DEVELOPMENT OF MECHANICAL ARM SUPPORT FOR SPINAL MUSCULAR ATROPHY PATIENT
}

\author{
Dedy H.B. Wicaksono1,*, Daniel J. Engel ${ }^{1}$, Leticia A. Genilar'1, Samantha T. Wijaya1, Samuel A. \\ Setiawan $^{1}$, Xaviera Jovinka ${ }^{1}$, and Lydia A. Kidarsa ${ }^{2}$ \\ ${ }^{1}$ Department of Biomedical Engineering, Faculty of Life Sciences and Technology, Swiss German University (SGU), \\ Tangerang 15143 \\ ${ }^{2}$ Bioteknik Design, Bandung \\ *corresponding author's email: dedy.wicaksono@sgu.ac.id; dedy.wicaksono@gmail.com
}

\begin{abstract}
Spinal Muscular Atrophy or SMA disorder is generally thought to have affected as many as 1 in 40 in country like USA. SMA type 2 and 3 are commonly found in Indonesia. Children who suffer from SMA disease cannot move their hands with flexion - extension and abduction - adduction orientation maximally, because the muscles in the patient cannot support the movements of the hand. In this project, an initial joint effort was conducted by SGU and Bioteknik Design, to develop an active mechanical arm support with muscular feedback. Hence, the hand movements of the patient can be supported externally through detecting the muscle tension produced by moving the patient's hand. The tension is detected using Carbon Nanotube (CNT)-coated thread Mechanomyography (MMG) sensor. The sensor signal is then fed into Arduino microcontroller, to give appropriate control signal to the universal power window motor. As an early proof of concept, wood was used as the main structural material for the arm support. The project, however, did not go as expected due to the lack of torque from the motor and missed feedback connection from the sensor. A counter balance mechanism like spring may be attached for future improvement.
\end{abstract}

Keywords: Spinal Muscular Atrophy, Mechanical Arm Support, Mechanomyography (MMG), Universal Power Motor

\section{INTRODUCTION}

Spinal Muscular Atrophy or SMA disorder is generally thought to have affected as many as 1 in 40 Americans, or about 6 million people. SMA is an autosomal recessive genetic disease, meaning that the condition involves two copies of a defective gene. SMA carriers do not show SMA disease symptoms, but carry a copy of the abnormal SMN1 gene. If both parents are carriers of SMA genes, then each child has one in four chances of developing the disease. There are four different types of SMA disease, type 1 to type 4 . Type 2 and
Type 3 were commonly found in Indonesia, according to Dr. Dian Kesumapramudya Nurputra, $\mathrm{PhD}$ from Sardjito Hospital, Yogyakarta. Epidemiologically, the majority of people suffered from Type 2 and Type 3.

Children who suffer from SMA disease cannot move their hands with flexion - extension and abduction - adduction orientation maximally, because the muscles in the patient cannot support the movements of the hand. By using this "mechanical arm support," the hand movements of the patient can be supported externally through detecting the muscle tension produced by moving

$$
\text { Nama Sub tema Penelitian }
$$


the patient's hand. If the prototype of this tool can be developed, then this tool can be applied to help SMA sufferers and improve patient mobility.

\section{MATERIAL AND METHODS \\ 1. Equipment and Materials}

These are the components and equipment used for developing the Mechanical Arm Support. 1) Arduino Uno. Arduino Uno is a Microcontroller board that uses ATmega328 controller chip and can be used to do almost any functions that a micro controller should do. 2) A 12-V universal power window motor. Universal power window motor is commonly used for car windows. It uses a gear mechanism to increase its torque by using a worm gear to rotate a bigger gear that attached to a smaller gear which is the output gear that can produce $3 \mathrm{Nm}$ of torque. 3) 12V Relay. The 12-volt relay are used to power the $12 \mathrm{v}$ universal power window motor, due to the Arduino only manage to produce 5 volts. 4) Bread board. The bread board are used to form the circuits for the electrical component of the project by using resistors, cables, switch and etc. 5) Wooden plank. It is the main material to make the prototype, since it is cheaper than steel, light weight and it has quite high tensile strength depending on its density. 6) Infra-board. Infraboard is a plastic board that has square cavity in the middle of the plane and it is very light. 7) Solidworks. Solidworks is a mechanical 3D CAD (Computer Aided Design) it is used for its capability to create the 3D Model complete with its structural density and weight. 8) Blender. Blender is a $3 \mathrm{D}$ graphics program that could made a 3D model and animation of it, it is a free alternatives of solid works despite the lacking of physics for the Solidworks. 9) Arduino. Arduino is a software that are used to control the Arduino Uno it used simplified C languages with the help of few libraries from Arduino.

\section{Sensor Placement and Preparation}

First, a slim fit-shirt will be worn by one of the subject to mark the sensor placements according to the morphology of their muscles that will be used. The CNT thread muscular activation sensor (Wicaksono, 2018) was sewn on with the pattern as in Fig 1. The remaining thread was tied to each other at the back of the cloth in order to make the resistance of the thread become smaller and increasing the strain of the thread. After the CNT thread was sewed then the thread will be covered with another fabric to prevent friction between the thread and the body which may affect the resistance of the material thus affecting voltage change. These sensors will receive signals from the muscles in form of vibration and proprioception that will become inputs to drive the two motors on the system.

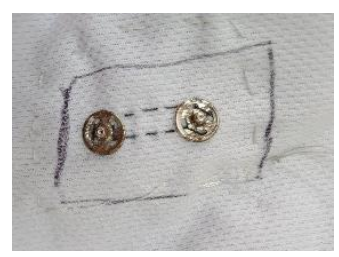

Figure 1. CNT-coated thread mechanomyography (MMG) sensor sewn pattern

The characteristic of whether the voltage will decrease or increase depends upon the condition of the thread during the action performed. The CNT will decrease in in resistance when strain is given, and increases when they are loosen. This is mostly done due to the proprioception on the subject, which is why the strain of the thread was increased between each electrode, to increase sensitivity but also too keep it stable.

\section{Driving Mechanism}

Driving mechanism is the program to activate the device to turn clockwise or anticlockwise. The main materials used for this section are Arduino Uno, four relays, and two motors. The three devices were interfaced with one another using external power supply of $12 \mathrm{~V}$ to run the motor. Four relays of the same type were used due to each motor requires two relays to run it. The first two relays function to turn the motors clockwise, while the other two relays function to turn the motors counterclockwise.

The trial period of using relay to activate the motor was using LED. The trial uses relay that was interfaced with LED. Switch buttons were also used for the LED program testing. The objective

$$
\text { Nama Sub tema Penelitian }
$$


was to turn on the LED once the button was pushed, and turn off the LED once the button was released.

\section{LED trial schematic}

During the trial program, LED was used. All pins of the relay were interfaced to the Arduino (figure 2). The Vcc pin of the relay was connected to $5 \mathrm{~V}$ pin of Arduino. The GND pin of the relay was connected to GND pin of Arduino. The IN pin of the relay was connected to the input pin of Arduino. In this case, the input connection of the Arduino was pin 3.

The relay has 3 other pins that function as normally open (right), com (middle), normally close (left). The normally close pin was not connected to any source. This is due to the purpose of making the normal state of the LED to be 0 (low/off). The normally open pin was connected to the $5 \mathrm{~V}$ pin of Arduino, so that when the com pin switched to the normally open, the state of the LED turned to be 1 (high/on).

Another pin of Arduino was used for the button function. This button function will determine the movement of the relay. The $5 \mathrm{~V}$ pin of the Arduino was connected to a resistor. The other side of the resistor was then connected to the button, while the other side of the button was connected to the GND pin. The connection of the button with the Arduino was between the resistor and the button. In this program, the used pin of the Arduino was pin 6. Pin 6 will read the state of the button to determine the desired time to activate the relay. Thus, the high state of the LED was the result of the working program.
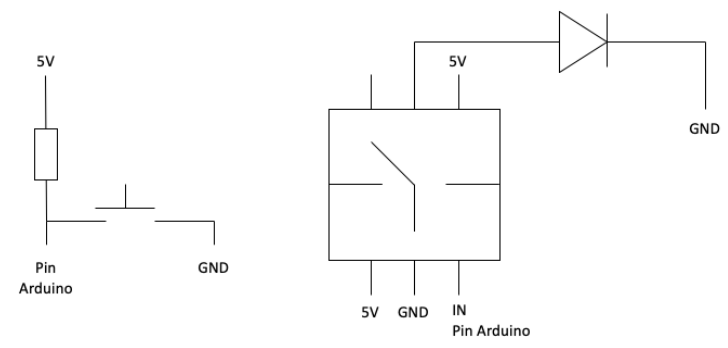

Figure 2. Schematic of LED testing circuit

5. Motor trial schematic
After the trial with the LED succeeded, then the trial to activate the motor was the next step. Two relays were used to activate the motor. One relay functions to rotate the motor clockwise, and another relay functions to rotate the motor counterclockwise. The schematic of the circuit has similar connection. However, there are several changes of the relay connection (figure 3 ).

The normally close and normally open pin of the relay was connected to an external power supply of $12 \mathrm{~V}$. This is due to the motor requires $12 \mathrm{~V}$ input to activate it. Therefore, the connection of with the motor was with the com pin of the relay.

In the previous trial, a button was used to activate the system. In this trial, potentiometer was used. The used of potentiometer was in purpose of representing the state of the sensor that will be used to detect the muscle activation of the child. The program will read the voltage of the potentiometer. Thus, the motor will be activated in the certain range of the determined voltage, and the motor will stop rotating when the voltage does not in the range of the determined voltage.
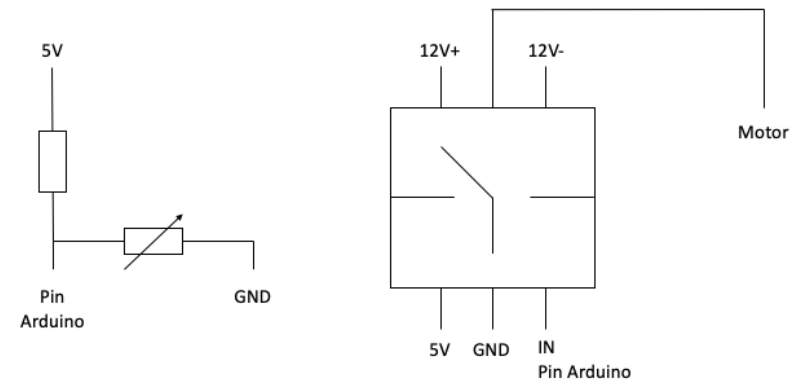

Figure 3. Schematic of motor testing circuit

\section{The Musculoskeletal system}

The human body has 2 different groups of muscle that are activated during a movement or an intention of movement, namely the effector and fixator. In this study, the specified movements and position are anterior elevation (flexion) and lateral elevation (abduction). This can be done by identifying a common muscle that is both activated during the 2 movements as a reference parameter.

The 3 muscle group that was chosen are Anterior Deltoid (AD), Lateral Deltoid (LD), and Seratus Anterior (SA). This is due to the contraction caused by the SA that acts as both the effector and 
fixator during both movements. AD acts for flexion and LD for abduction (fig. 4).

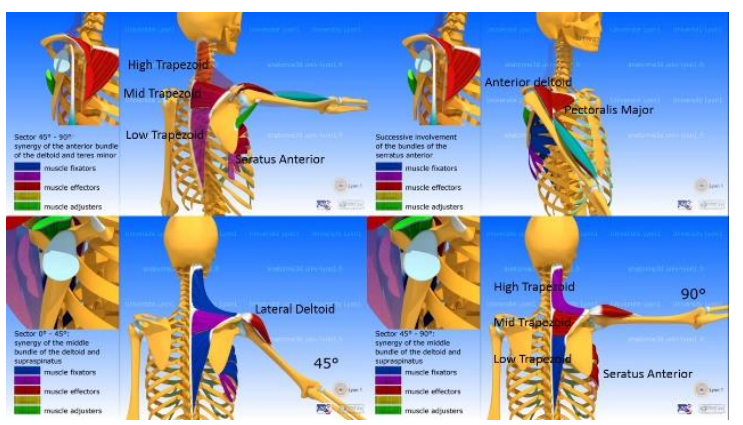

Figure 4. Muscular activity during flexion and abduction motion (3D Anatomy Lyon 2014a, 2014b)

In lateral elevation, during the $0^{\circ}-45^{\circ}$ elevation, the mid Trapezoid and SA acts as the fixator then it begins to act as an effector. During the transition from $45^{\circ}-90^{\circ}$, mid Trapezoid and SA becomes the effector. In anterior elevation from $0^{\circ}$ $45^{\circ}, \mathrm{SA}$ acts as the fixators and $\mathrm{AD}$ is the effector. The transition from $45^{\circ}-90^{\circ}$ SA start changing into an effector while the AD remains as the effector.

The muscle noises are caused due to the actomyosin filaments on the skin to expand due to contraction, thus, shortening the filaments and produce the so called vibration from the muscle. The deltoid has similar noise produced with the bicep which is in a range between $5 \mathrm{~Hz}-100 \mathrm{~Hz}$ or $5 \mathrm{~Hz}-250 \mathrm{~Hz}$ according to several studies (Hendrix, 2010, Armstrong, 2010, Tanaka, 2011, Oster, 1980).

\section{Data taking Protocol}

The data taking protocol involves seven different positional class which are: Rest, Rest-toFlex, Flexion, Flex-to-Rest, Rest-to-Abduct, Abduction, and Abduct-to-Rest. Each position and movement are done for 8 seconds followed by another 8 seconds of rest afterwards to obtain the baseline again. The movements are not done consecutively, but rather separately for both abduction and flexion.

\section{MMG Sensor Code and Circuit}

The MMG sensor code is written using $\mathrm{C} / \mathrm{C}++$, in Arduino IDE. The code utilizes a digital Infinite Impulse Response (IIR) low pass filter library created by Martin Vincent Bloedorn (Bloedorn, 2019) with the cutoff frequency at 150 $\mathrm{Hz}$. The sampling frequency is set to $160 \mathrm{~Hz}$, and utilizes both the analog and digital pin as detector and trigger respectively. The circuit used is a combination of a voltage divider and an amplifier with adjustable gain.

\section{The Code}

The digital filter used in this study is a second order low pass filter that has a cutoff frequency at $150 \mathrm{~Hz}$. This is done to isolate the external noise cause by the area surrounding the muscle of interest. The higher the order of the filter, the sharper the cutoff will be. The Arduino Baud Rate is put at 115200 in order to be able to read the filtrated value from the sensor.

The signal variables has 3 inputs set (AD, $\mathrm{LD}, \mathrm{SA}$ ) which are connected to the analog pin and have a maximum output of $5 \mathrm{~V}$. it is then combined with the relay controller module to control the motor by creating an IF/ELSE condition in the Loop() function that satisfy the condition for an intention of a movement.

\section{The Circuit}

The CNT resistance will be controlled to be lower than $100 \mathrm{k} \Omega$ to maintain stability and ratio of voltage division. The input signal will first go through a voltage divider with the reference resistance of $1 \mathrm{M}$ (mega) $\Omega$ (fig. 5).

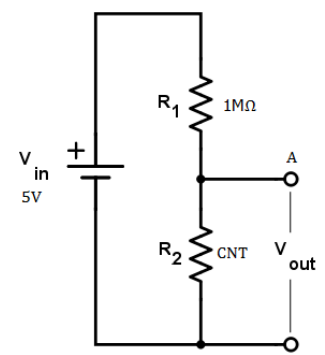

Figure 5. Voltage Divider Circuit (A; connects to amplifier circuit) 
Then it will be followed by an amplification using TL072CP due to its low-noise characteristic (Futurlec datasheet, 2020). This circuit is a noninverting amplifier circuit that uses $10 \mathrm{k} \Omega$ potentiometer as the reference resistor alongside a $100 \mathrm{k} \Omega$ resistor (Fig. 6), control the gain from the range of 1 to $11 \mathrm{x}$ amplification the output from the amplification will be connected to the analog pin of the Arduino for the value to be read.

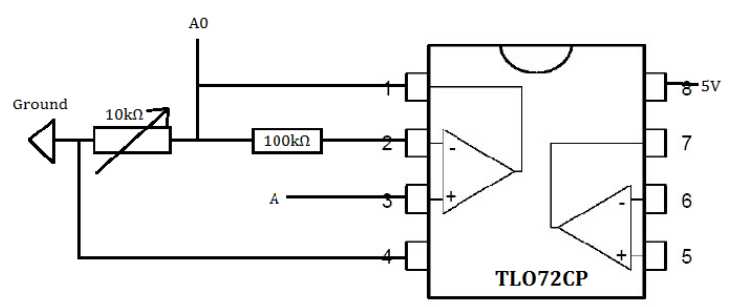

Figure 6. Amplifier Circuit (A0; to Analog pin Arduino, A; from Voltage divider circuit)

\section{RESULTS AND DISCUSSION}

\section{MMG Results}

The total number of data collected on average for 1 trial is around 6450 samples, in which for each action has 3 trials. It can be seen from the graph shown in Figure 7, that there are significant voltage change that occurred during the movements and positional changes accompanied with an increase in ripple on the full range of motion (abduction and flexion).

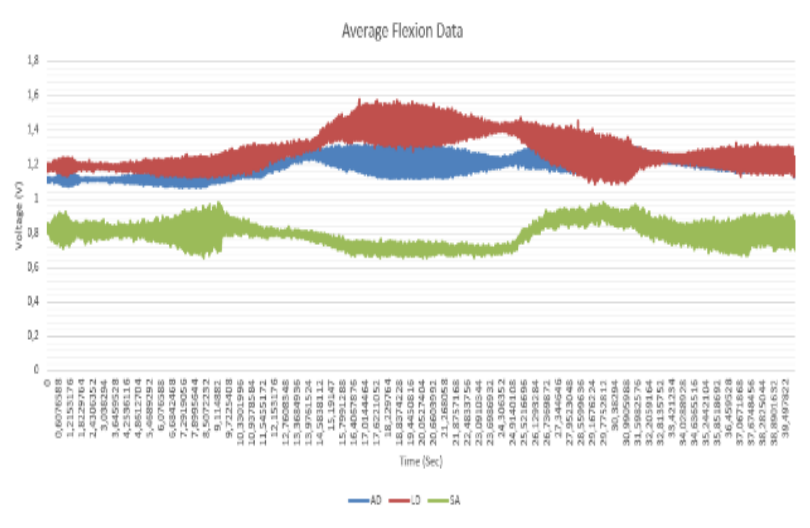

Figure 7. Average Abduction Data (V - Time)

The data is classified per positional changes according to time, such that, (1) Rest, $0-8$ seconds;
(2) Rest-to-Abduct, 8 - 16 seconds; (3) Abduction, 16 - 24 seconds; (4) Abduct-to-Rest, 24 - 32 seconds; (5) Rest, 32 - 40 seconds. Similar like the case with the previous flexion data, the displayed result is the average value from 3 trials. The Red label represents potential difference at LT, the Blue represents the potential difference at $\mathrm{AD}$ and the Green represents the potential difference at SA.

The result displayed a similar result to the Flexion data but with a larger significance at the maximum potential difference between sensors where LD experienced the maximum difference of $0.32 \mathrm{~V}$ and $\mathrm{AD}$ experience $0.02 \mathrm{~V}$ change, and $\mathrm{SA}$ experience $0.09 \mathrm{~V}$ change (from the difference between values shown in Table 1 and Table 2).

During the full abduction motion, it can be seen that the ripple of the LD data has increased dramatically which indicated vibration of muscle due to tension and moment at the deltoid.

By extracting and averaging the data during the full range of motion of Flexion and Abduction, the minimum potential difference that acts as the activation or initiation during muscle intention to move can be obtained.

Table 1. Flexion Average Potential difference for Muscle activation

\begin{tabular}{|c|c|c|}
\hline \multicolumn{3}{|c|}{$\begin{array}{l}\text { Average Potential difference for } \\
\text { Muscle activation }\end{array}$} \\
\hline $\mathrm{AD}$ & LD & SA \\
\hline 1.218478 & 1.424692 & 0.708403 \\
\hline
\end{tabular}

Table 2. Abduction Average Potential difference for Muscle activation

\begin{tabular}{|c|c|c|}
\hline \multicolumn{3}{|c|}{$\begin{array}{l}\text { Average Potential difference for } \\
\text { Muscle activation }\end{array}$} \\
\hline AD & LD & SA \\
\hline 0.964713 & 1.162922 & 0.604425 \\
\hline
\end{tabular}

Using similar method, the average potential energy during resting period can also be calculated, thus, obtaining the baseline of each of the respective signal, as shown in Table 3 and Table 4.

$$
\text { Nama Sub tema Penelitian }
$$


Table 3. Flexion Average Potential Difference at Rest

\begin{tabular}{|c|c|c|}
\hline \multicolumn{3}{|c|}{ Average Potential difference at } \\
Rest \\
\hline AD & LD & SA \\
\hline 1.165298 & 1.209737 & 0.80884 \\
\hline
\end{tabular}

Table 4. Abduction Average Potential Difference at Rest

\begin{tabular}{|c|c|c|}
\hline \multicolumn{3}{|c|}{$\begin{array}{c}\text { Average Potential difference for } \\
\text { Muscle activation }\end{array}$} \\
\hline AD & LD & SA \\
\hline 0.942226 & 0.839902 & 0.690644 \\
\hline
\end{tabular}

Therefore, by adjusting the value as the parameter in the Arduino program, it is possible to control the mechanical arm support as long as the condition satisfy the parameter.

However, it is important to note that these data has not been normalized properly. Moreover, the data has only been taken from 1 subject, which indicates the possibility that different subject may have different data characteristic since the physical morphology of every people differ.

Furthermore, the rotation of the motor cannot be controlled to stop at a specific angle after the first activation. This is because the subject is performing the movement and holding the position, the muscle is always contracting rather than being relaxed as it is intended for the SMA patient (as an arm support), and therefore the motor keeps rotating as it is unable to detect the relaxed muscle.

From these data, it can be seen that both are very similar in terms of characteristic and muscle activation group. This will make differentiation between the two motion difficult and more sensors will be needed. More subject need to be tested in order to test the coherency of this data characteristic.

For future improvement, it is important to set a baseline for the CNT thread resistance and proper calibration technique and a modification towards the data taking procedure to accurately mimic the SMA patient characteristic where only the intention of the movement is detected rather than the full proprioception.

\section{Mechanism}

The mechanism used in this project is by creating 3D pivoting mechanism by using 2 motors. One is for the lateral movements; abduction, adduction. The second one would be for flexion and extension of the arm. The motors was placed in the back of the shoulder for the lateral movements, while supporting the other motor. and in the side of the shoulder for the flexion and extension (Fig. 8).

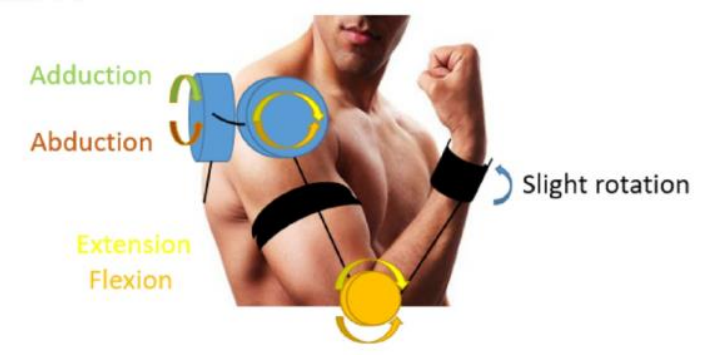

Figure 8. Illustration of the mechanism.

Assuming the length of the arm are $60 \mathrm{~cm}$. the weight of the arm is $2 \mathrm{~kg}$ and the weight of the motor is $1 \mathrm{~kg}$ here is the illustrations for the how much torque needed for the motor at the heaviest moment possible (without lifting any additional weights).

The total torque needed is calculated as follows $(8.929 \mathrm{Nm}+0.495 \mathrm{Nm}) \times \sin 90^{\circ}=9.308$ $\mathrm{Nm}$ is needed to lift the arm from the lateral motor at its heaviest moment possible (Fig. 9 and 10).

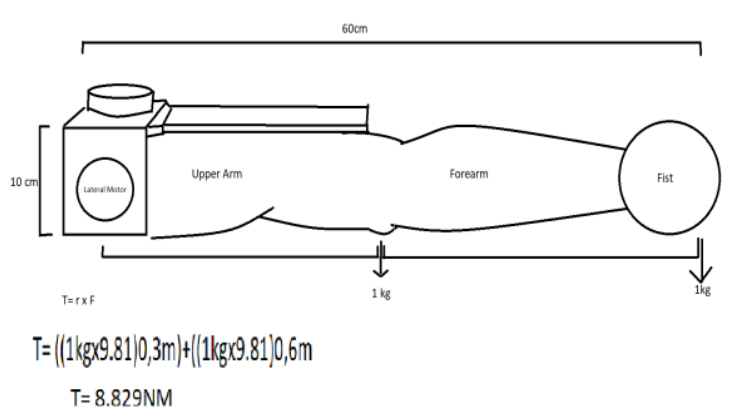

Figure 9. Illustration of the torque needed for the lateral motor to lift the arm in the heaviest possible moment. 


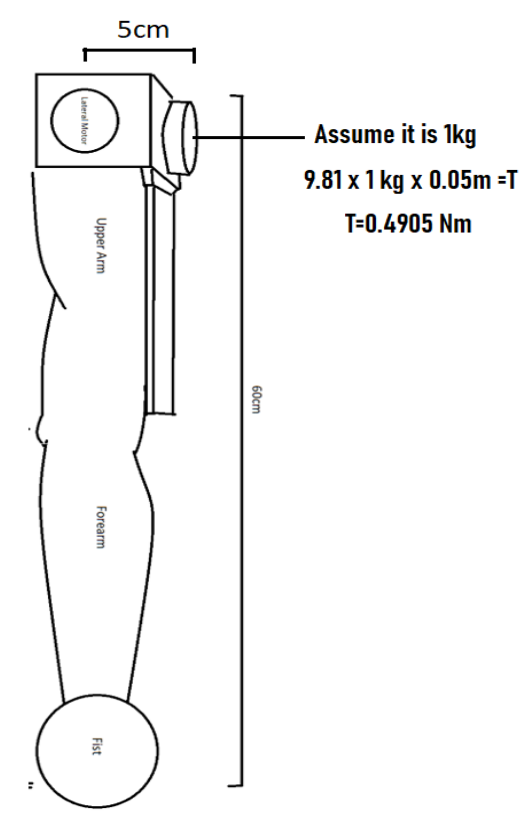

Figure 10. Illustration of the torque needed for the lateral motor to lift the motor in the heaviest moment.

\section{Design}

The first design is using NEMA 17 motor, the motor was considered to be most suitable, because of its customizable factor and can easily be found in the market therefore we can design it with our needs. But the final product is using power window, the motor has a higher torque compared to the NEMA 17.To design the arm support, Solid Works software was used. This platform is widely used to design machines or parts that can be customized based on our needs. There are few highlights criteria to design the arm support. The main problem is to make the arm support as light and slim as possible but in the same time still able to move the arm without damaging it. The design are shown in Fig. 11, 12, and 13.

\section{Trial Results}

The arm support main material is using $1 \mathrm{~cm}$ thick wood plate and infra-board jointed using an L bar and the rotor of the motor and it weight $2.3 \mathrm{~kg}$. but because there is no coupling system available for the gear so the motor was simplified into a dynamo motor so it could be mounted to the rest of the arm support.

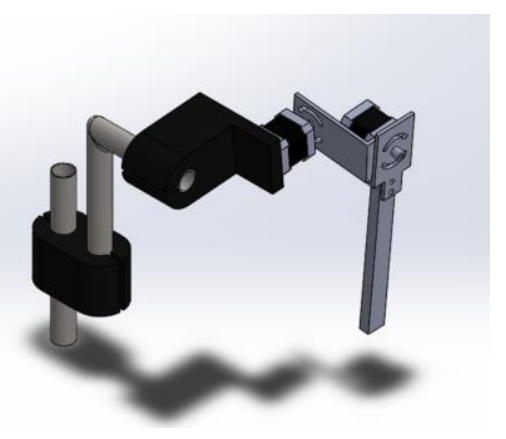

Figure 11. Arm support design with NEMA 17 and chair mounting system using solid works.

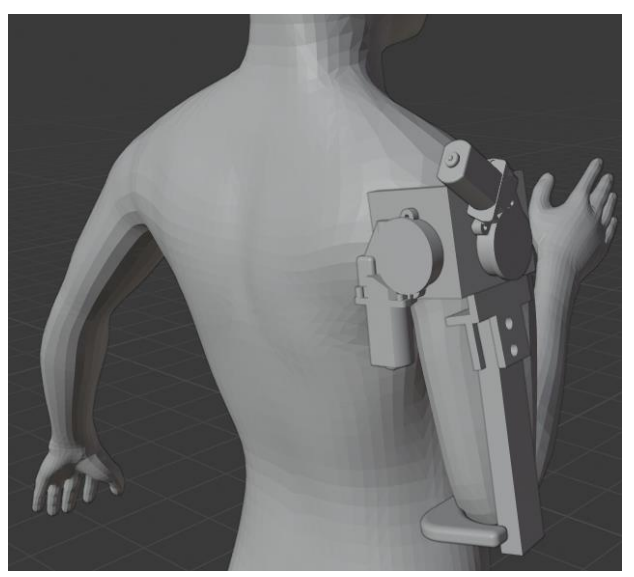

Figure 12. Arm support design with universal power window using Blender

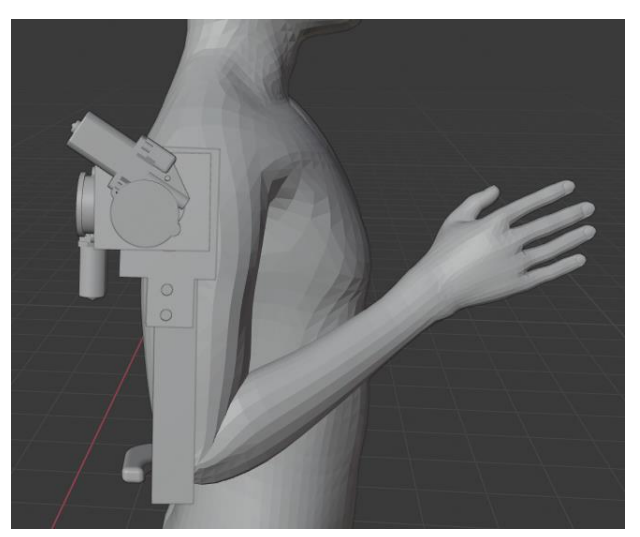

Figure 13. Arm support design with universal power window using Blender

Nama Sub tema Penelitian 
The problem occurs when designing the coupled system for the power windows. The gear system is only suitable for windows inside cars it using 7 edge gears with unique star shape, and finding the coupled system to be attached to the design would be difficult to obtain and expensive to create using steel. Although we manage to create a coupling system for the flexing and extension movement part of the arm support using toothpicks and plastics it is not applicable for the lateral movement motor because the coupling system that has been made is too fragile (Fig. 14).

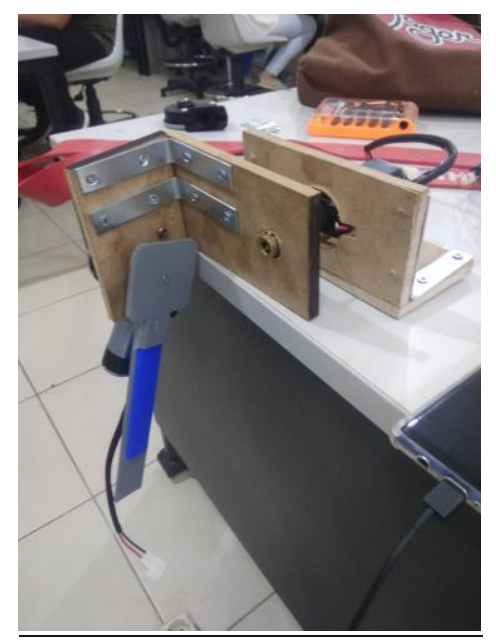

Figure 14. Arm support design with universal power window proof of concept model

After designing and creating the proof of concept turns out that the high torque power window motor for the lateral movements was not able to withstand the weight of the other motor, so it was mounted on a wooden plate to be mounted on the table (Fig. 14).

The proof of concept model manages to move the arm support on the flexing and extension movement using inputs from the muscle stimulation captured by the CNT mechanomyography as shown in Figure 15.

In the future, to solve the problem of high torque power requirement, a spring with pulley and spring can be added to hold the weight of the arm. Hence, the motor's torque is only required to change the arm's position.

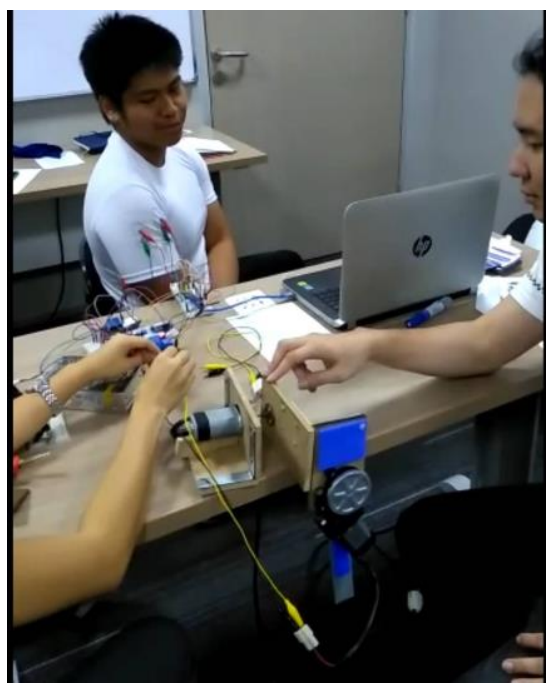

Figure 15. Arm support design with universal power window proof of concept moved by using inputs from CNT mechanomyography

\section{Budgeting}

Table 5. Budgeting of the project

\begin{tabular}{|l|r|}
\hline Item Names & \multicolumn{1}{l|}{ Price } \\
\hline $\begin{array}{l}\text { Jumper Cable arduino male to } \\
\text { female }\end{array}$ & $\mathrm{Rp28.000}$ \\
\hline $\begin{array}{l}\text { Universal motor power window 3 } \\
\text { holes 7 sided gear }\end{array}$ & $\mathrm{Rp} 187.000$ \\
\hline Dry fit Shirt & $\mathrm{Rp} 38.000$ \\
\hline $\begin{array}{l}\text { Relay Module 12 Volt 2 Channel } \\
\text { 12v }\end{array}$ & $\mathrm{Rp53.000}$ \\
\hline Triplex board & $\mathrm{Rp} 110.000$ \\
\hline Total Price & $\mathrm{Rp} 416.000$ \\
\hline
\end{tabular}

This project manages to cut down the budget by using few parts that are already available in the lab and by using wood, it is cheaper and easier to form rather than steel.

\section{CONCLUSION}

In conclusion the proof of concept model can be categorized as a failure. Despite the lateral motor manage to move the arm support a bit, it cannot hold the rest of the arm support and it need 
to be simplified into a simple dynamo motor to be mounted with the arm support. as for the extension and flexion motor were able to move the rest of the arm support because the material is used for the rest of it was infra board which is considerably light. But this project manages to capture the inputs from the muscles to move the flexion and extension part of the arm support.

The motors failed to move the arm support properly because it only has $3 \mathrm{Nm}$ of torque using the gear mechanism. In this project the gear mechanism was not used. It only uses the raw power of the dynamo a third of the power while using gear mechanism, which is roughly $1 \mathrm{Nm}$. According to the calculation the dynamo should be able to rotate the Lateral movements of the arm support because the torques required to lift the arm support with its motor are $0,49 \mathrm{Nm}$. However, the dynamo cannot turn the lateral movements because the rotor was dragged down a bit due to the weight of the arm support, which moving its axis from the magnetic field of the coil disrupting the magnetic field of the coil inside the dynamo resulting significant loss of torque.

The motor power window was a DC motor which is only activated if the motor gets the electricity, so it could not maintain its position if there are no more inputs given to the motors, but by using the power window it could manage holding its position by using it sheer force of the gear torque. However, if the weight ha a higher force than the torque it won't keep its position. The other weakness of using the power window is there are no feedback, it could be fixed by using a driver, but nevertheless because there is no feedback, the motor can spin $360^{\circ}$ and it will not know its actual position of the arm support and the speed could not be controlled.

For further improvements a counter balance mechanism or a spring attached to the Arm support are required to help and support the motors moving the arm support. Change the motor into high torque Stepper motor it rotate per steps. So the speed can be controlled while knowing the arm support actual position by counting the steps or using a driver or encoder to have an active feedback of the motor.

\section{REFERENCES}

3D Anatomy Lyon. (2014) "Anterior elevation of the arm and synergies of the rotator cuff." YouTube, https://www.youtube.com/watch?v=fDW5eO593N $\underline{\mathrm{w} \& \mathrm{t}=31 \mathrm{~s}}$

3D Anatomy Lyon. (2014). "Lateral elevation of the arm in the plane of the scapula." YouTube, https://www.youtube.com/watch?v=LKo5hlD6GW $\underline{8}$

Armstrong et al. (2010), "Reliability of mechanomyography and triaxial accelerometry in the assessment of balance", Journal of Electromyography and Kinesiology, (20) 726-731. Bloedorn, $\mathrm{M}$. (2019) "MartinBloedorn/LibFilter." GitHub, https://github.com/MartinBloedorn/libFilter.

Hendrix et al. (2010). "Comparing electromyographic and mechanomyographic frequency-based fatigue thresholds to critical torque during isometric forearm flexion." Journal of Neuroscience Methods, 194(1). 64-72.

Oster, Gerald, and Joshua S. Jaffe. (1980) "Low frequency sounds from sustained contraction of human skeletal muscle." Biophysical journal 30(1). 119-127.

SMA Foundation. "About SMA: Overview" .https://smafoundation.org/about-sma/.

Tanaka et al. (2011). "Study on evaluation of muscle conditions using a mechanomyogram sensor," Systems, Man, and Cybernetics (SMC), 2011 IEEE International Conference on, vol., no., pp.741-745, 9-12 Oct. 2011.

"TL072CP - TL072 Low Noise JFET Dual Op-Amp." Futurlec,

Wicaksono, D. H., Benhanan, B., Parung, D. E., Ughi, F., Shafi, A., Mandala, S., ... \& Yuliarto, B. (2018). Carbon Nanotube-Coated Thread as Sensor for Wearable Mechanomyography of Leg Muscles. In 2018 IEEE SENSORS (pp. 1-4). IEEE. 